\title{
Incontinencia de esfuerzo: Experiencia quirúrgica en nuestro centro
}

\author{
Torres Zambrano G, Lujan Galán M, Martín García C, García Tello A, Rodríguez N, \\ Berenguer Sánchez A.
}

Servicio de Urología. Hospital Universitario de Getafe. Madrid.

Actas Urol Esp. 2007;31(8):858-862

\section{RESUMEN}

INCONTINENCIA DE ESFUERZO: EXPERIENCIA QUIRÚRGICA EN NUESTRO CENTRO

Introducción: Para el tratamiento quirúrgico de la incontinencia urinaria de esfuerzo existen múltiples técnicas, en la actualidad la de referencia es la descrita por Burch. Comparamos los resultados globales y de cada técnica en cuanto a las tasas de continencia postoperatoria y de complicaciones observadas en nuestra experiencia.

Materiales y métodos: Hicimos un análisis retrospectivo de 292 mujeres operadas por primera vez por presentar incontinencia urinaria de stress en nuestro Centro, entre 1991 y 2006. Consideramos a las pacientes curadas si no presentaban ningún grado de incontinencia postoperatoria, se realizó un análisis de Kaplan - Meier para la evaluación de la continencia postoperatoria. Además estudiamos el comportamiento de las complicaciones postoperatorias presentadas.

Resultados: La media de edad de las pacientes fue de 53,6 años, con un tiempo de seguimiento medio de 15,6 meses. La mayoría de las intervenciones fueron técnicas vaginales (138), seguida por los TVT (57), Burch abdominal (38), Sling con parche de vagina (33), y la menos utilizada fue el Burch laparoscópico (26). Las mejores tasas de continencia postoperatoria a los dos años se consiguieron con el TVT $(87,6 \%)$, luego con el Sling con parche de vagina $(84,9 \%)$, las siguientes con Burch laparoscópico $(59,3 \%)$, Burch abdominal $(59,1 \%)$, y las peores con técnicas vaginales $(48,2 \%)$, ( $\mathrm{p}=0,007)$. Se observó mayor urgencia y urgencia-incontinencia postoperatorias en las pacientes operadas mediante TVT $(36,8 \%$ y $21,1 \%$ respectivamente), y mayor dolor postoperatorio tras las técnicas vaginales $(36,2 \%)$. La retención urinaria postoperatoria y residuo postmiccional elevado más comunes entre el Sling con parche de vagina $(54,5 \%)$ y las técnicas vaginales $(26,1 \%)$ respectivamente.

Conclusiones: La técnica con mejores resultados para manejo de incontinencia en nuestra población es el TVT.

Palabras clave: Incontinencia urinaria de esfuerzo. Cirugia de incontinencia. Complicaciones quirúrgicas.

\section{ABSTRACT \\ URINARY STRESS INCONTINENCE: SURGICAL EXPERIENCE IN OUR CENTER}

Introduction: For the treatment of the urinary stress incontinence, there are many techniques, and today the gold standard is that described by Burch. We compared the global and each technique results about rates of postoperative continence and complications seen in our experience.

Materials and methods: We made a retrospective analysis of 292 women that have the first surgery for urinary stress incontinence in our centre, between 1991 and 2006. We considered cure the patient who did not have any level of incontinence postoperative, we made a Kaplan - Meier analysis for the postoperative continence evaluation. Besides we studied the behaviour of the postoperative complications found.

Results: Mean age was 53.6 years old, and mean time of follow up was 15.6 months. The vaginal techniques were the most practised (138), followed by the TVT (57), abdominal Burch (38), vaginal wall Sling (33), and the less was the laparoscopic Burch (26). The best rates of postoperative continence after 2 years were observed in the patients treated by TVT $(87,6 \%)$, then with vaginal wall Sling $(84,9 \%)$, laparoscopic Burch (59.3\%), abdominal Burch $(59,1 \%)$, and the worst with vaginal techniques $(48,2 \%)(p=0,007)$. We found more urgency and urge incontinence in the patients treated by TVT (36,8\% y $21,1 \%$ respectively), and postoperative pain with vaginal techniques $(36,2 \%)$. Postoperative urine retention and postoperative high residual urine more with the vaginal wall sling $(54,5 \%)$ and vaginal techniques $(26,1 \%)$ respectively.

Conclusions: We have the best results for the incontinence treatment in our population with the TVT technique.

Keywords: Urinary stress incontinence. Incontinence surgery. Postoperative complications. 
$\mathrm{L}^{\mathrm{a}}$ a incontinencia urinaria es un problema de salud que tiene un gran impacto sobre la calidad de vida de las personas que la padecen. Se estima la prevalencia en España en dos millones de personas, de las que un 50\% son mujeres mayores de 60 años $^{1}$. La incontinencia urinaria de esfuerzo se define por la Sociedad Internacional de Continencia como una perdida involuntaria de orina coincidiendo con el aumento de presión intra-abdominal en ausencia de contracciones del detrusor o una vejiga sobredistendi$\mathrm{da}^{2}$. Para su tratamiento existen descritas múltiples técnicas desde que Kelly y Dumm en el año de 1914 publicaron los resultados de la plicación uretral $^{3,4}$, hasta nuestros días, siendo en la actualidad la técnica de referencia la descrita por Burch en $1961^{5,6}$. No obstante, técnicas como los cabestrillos, TVT y TOT están aumentando su acogida por los buenos resultados y la seguridad asociados ${ }^{7}$, aun que las series disponibles no aportan seguimiento suficiente ${ }^{3}$.

Es necesario tener en cuenta que la incontinencia urinaria mixta tiene menores tasas de éxito postoperatorio $^{5}$ que la incontinencia de estrés pura. Por ello es importante una adecuada valoración de los síntomas en la consulta ambulatoria, ya que los sintomas de urgencia responden a tratamientos médicos no invasivos y hay que diferenciarlos de una urgencia-incontinencia de novo postoperatoria $^{8,9}$. Además, ninguna de las técnicas empleadas está exenta de complicaciones ${ }^{8}$.

El objetivo del presente trabajo es comparar los resultados globales y de cada técnica en cuanto a las tasas de continencia postoperatoria y de complicaciones observadas en nuestra experiencia.

\section{MATERIALES Y METODOS}

Se llevó a cabo un análisis retrospectivo de 292 mujeres operadas por primera vez por presentar incontinencia urinaria de stress. Recogemos la experiencia de nuestro Centro entre Julio de 1991 y mayo de 2006. Para facilitar el análisis hemos agrupado las distintas técnicas empleadas en

1) Cirugias vaginales (Raz I-II, Leach),

2) Abdominales (Burch),

3) Sling (cabestrillo con parche de vagina),

4) Burch laparoscópico y 5) TVT.
Debido a la metodología empleada en este trabajo (análisis de supervivencia) hemos considerado a las pacientes como curadas si no presentaban ningún grado de incontinencia postoperatoria, y definimos fracaso el momento en el cual aparece incontinencia tras la cirugia, con cualquier tipo de pérdida involuntaria de orina con el esfuerzo, sea cual sea su severidad ${ }^{10}$, analizando los resultados globales de nuestra serie, posteriormente comparando las técnicas entre sí. Se realizó un análisis de supervivencia con curvas de Kaplan-Meier ${ }^{11}$, donde el evento producido se definió como la aparición de la incontinencia de estrés postoperatoria, recogiéndose la fecha de aparición de esta y el tiempo transcurrido desde la cirugia hasta la misma. Se calcularon la media y mediana del tiempo transcurrido hasta la aparición de incontinencia, así como la proporción de pacientes libre de la misma en cada intervalo de seguimiento. Posteriormente se compararon entre sí las curvas de supervivencia correspondientes a cada una de las técnicas analizadas mediante el test de Breslow.

Además estudiamos el comportamiento de las complicaciones postoperatorias presentadas en nuestra muestra, incluyendo dolor postoperatorio, la urgencia, incontinencia de urgencia, retención postoperatoria, residuo posmiccional elevado y necesidad de cateterismo intermitente.

\section{RESULTADOS}

La media de edad de las pacientes fue de 53,6 años, con una mediana muy similar $(53,3)$, con un mínimo de 26 años y un máximo de 78 años. El tiempo de seguimiento medio de la serie fue de 15,6 meses, error estándar 1,1. De los 307 procedimientos la mayoría (138) fueron técnicas vaginales, seguida por los TVT (57). En la Tabla 1 se ofrece la distribución de la población estudiada según técnica empleada, así como el tiempo de seguimiento de cada grupo. La técnica menos utilizada fue el Burch laparoscópico, con sólo 26 pacientes.

En la Figura 1 representamos la evolución de la continencia postoperatoria en la muestra global estudiada $(\mathrm{N}=292)$. La probabilidad de permanecer libre de cualquier grado de incontinencia a $\operatorname{los} 6$, 12,18 y 24 meses tras la cirugía fue del $82,3 \%$, $68,8 \%, 60,7 \%$ y $59,9 \%$ respectivamente. La mediana de tiempo hasta la aparición de incontinencia fue de 86,6 meses. 
Tabla 1. Distribución de las técnicas empleadas y tiempo de seguimiento (años).

\begin{tabular}{|c|c|c|c|c|c|c|c|}
\hline & \multirow[t]{2}{*}{$\mathbf{N}$} & \multirow[t]{2}{*}{ Media } & \multirow{2}{*}{$\begin{array}{c}\text { Error estándar } \\
\text { media }\end{array}$} & \multicolumn{2}{|c|}{ IC media (95\%) } & \multirow[b]{2}{*}{ Minimo } & \multirow[b]{2}{*}{ Máximo } \\
\hline & & & & Limite inferior & Limite superior & & \\
\hline Vaginal & 138 & 16,82 & 1,77 & 13,32 & 20,31 & 1,00 & 124,62 \\
\hline Abdominal & 38 & 18,32 & 4,04 & 10,13 & 26,51 & 1,00 & 106,09 \\
\hline Sling & 33 & 17,28 & 3,34 & 10,49 & 24,07 & 1,00 & 62,52 \\
\hline Laparoscopia & 26 & 15,06 & 3,01 & 8,87 & 21,25 & 1,22 & 59,10 \\
\hline TVT & 57 & 13,85 & 1,29 & 11,27 & 16,44 & 0,00 & 36,24 \\
\hline Total & 292 & 15,64 & 1,07 & 13,53 & 17,76 & 0,00 & 124,62 \\
\hline
\end{tabular}

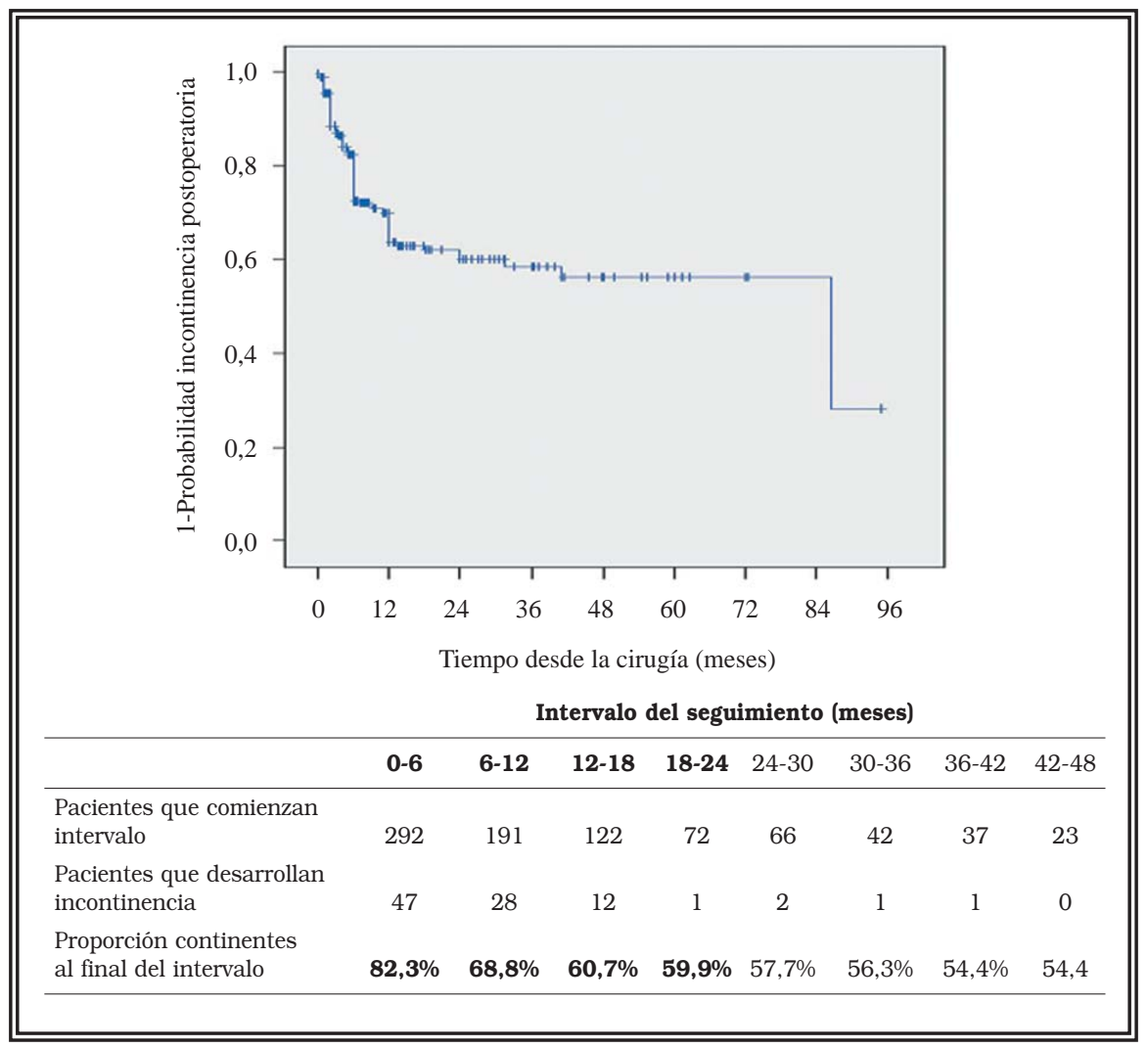

FIGURA 1. Evolución de la continencia postoperatoria. Toda la muestra ( $N=292)$. Análisis Kaplan-Meier. En la tabla acompañante se ofrece la probabilidad de permanecer libre de incontinencia según intervalo de seguimiento.
En cuanto al análisis de las complicaciones postoperatorias, se ofrecen de modo resumido en la Tabla 2. Se observó una mayor tasa de urgencia y urgencia-incontinencia postoperatorias en las pacientes operadas mediante TVT $(36,8 \%$ y $21,1 \%$ respectivamente). El dolor postoperatorio fue más frecuente tras las técnicas vaginales $(36,2 \%)$. La retención urinaria postoperatoria así como el residuo postmiccional elevado se observaron con más frecuencia tras las técnicas vaginales o de Sling con parche de vagina. Además, dos pacientes sometidas a corrección mediante TVT presentaron hematoma prevesical que requirió la retirada (parcial en un caso y total en otro) de la malla de polipropileno.
En la Figura 2 mostramos el análisis de los grupos de técnicas estudiados en cuanto a continencia postoperatoria.

Los mejores resultados en términos de continencia se observaron en las pacientes operadas mediante TVT, con un $87,6 \%$ totalmente libres de incontinencia de stress a los 2 años de seguimiento (Figura 1). Los peores resultados los ofrecieron las técnicas vaginales con 48,2\%. La diferencia entre las técnicas fue significativa (Breslow $\mathrm{p}=0,007$ ).

\section{DISCUSIÓN}

En el presente trabajo hemos incluido 292 pacientes operadas por primera vez para manejo de su incontinencia de stress. Tras analizar las distintas técnicas en cuanto a tasa de continencia postoperatoria, hemos encontrado los mejores resultados con el TVT (88\% totalmente continentes a los dos años), seguido muy de cerca por el Sling con parche de vagina (85\%), y el peor con las técnicas vaginales (48\%). 


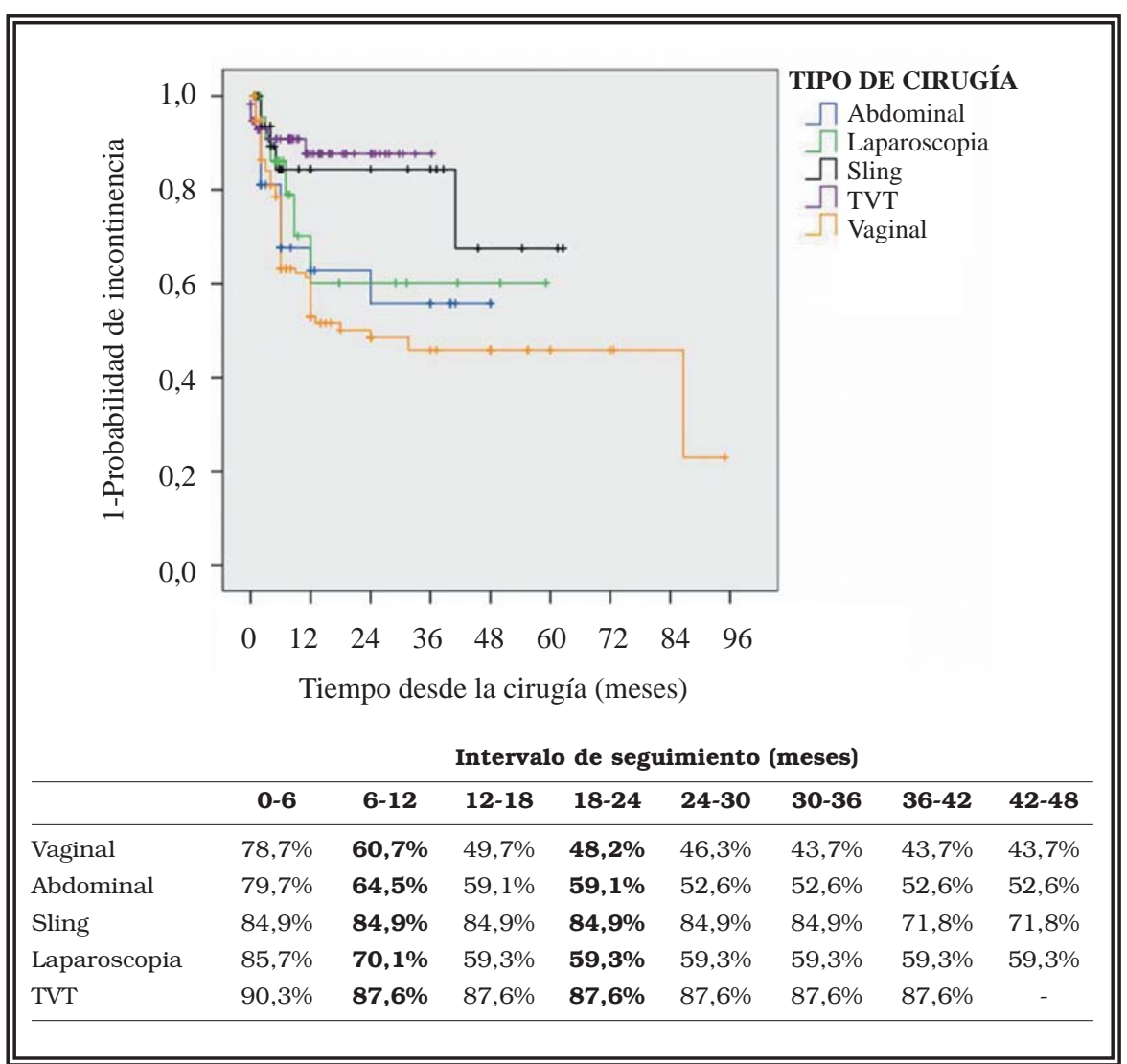

FIGURA 2: Evolución de la continencia postoperatoria. Desglose por técnicas. Análisis Kaplan-Meier. En la tabla acompañante se ofrece la probabilidad de permanecer libre de incontinencia al final del intervalo de seguimiento según tipo de técnica considerada.

Según una revisión de la Sociedad de Obstetras y Ginecólogos de Canadá (SOGC), los TVT demostraron en un corto plazo una equivalencia a los procedimientos retropúbicos tipo Burch, recomendando su práctica como primera cirugía para ofrecer a las pacientes, pero teniendo en cuenta que no existen estudios de equivalencias a largo plazo ${ }^{12}$.
Por otro lado, hemos encontrado tras el TVT una mayor tasa de urgencia $(36,8 \%)$ y urgencia-incontinencia $(21,1 \%)$ postoperatorias. Esta última es a menudo descrita en la literatura revisada, y se supone que es producida por una sobrecorrección asociada al uso de cintas de polipropileno elástico ${ }^{3}$. No obstante, aunque se ha reconocido a la obstrucción al vaciado vesical como la principal complicación reportada con el TVT $^{7,13}$, nosotros sólo la hemos encontrado en un $8,8 \%$ de nuestra serie. Además, 2 de nuestras 57 pacientes sometidas a TVT tuvieron que ser reintervenidas para drenaje de hematoma posquirúrgico $\mathrm{y}$ retirada de la malla de polipropileno.

En nuestra experiencia, las técnicas vaginales y el Sling con parche de vagina presentaron mayores porcentajes de dolor postoperatorio $(36,2 \% \mathrm{y}$ $27,3 \%$ respectivamente), retención postoperatoria $(26,8 \%$ y $54,4 \%)$, residuo posmiccional $(26,1 \%$ y $18,2 \%)$, todas ellas conocidas sobre todo en la literatura con el uso de Sling con parche de vagi$\mathrm{na}^{14}$. Además existen recomendaciones para no ofrecer las técnicas vaginales por su alta tasa de fallos ${ }^{12}$, impresión que también obtenemos tras el presente análisis $(48 \%$ a los dos años con esta

Tabla 2. Complicaciones postoperatorias observadas con mayor frecuencia tras cada uno de los grupos de técnicas quirúrgicas estudiadas. Test Chi-cuadrado.

\begin{tabular}{lccccccc}
\hline & Vaginal & Abdominal & Sling & Laparoscopia & TVT & Total & p \\
\hline $\mathrm{N}$ & $\mathbf{1 3 8}$ & $\mathbf{3 8}$ & $\mathbf{3 3}$ & $\mathbf{2 6}$ & $\mathbf{5 7}$ & $\mathbf{2 9 2}$ & \\
Urgencia & $1(0,7 \%)$ & $0(0 \%)$ & $5(15,2 \%)$ & $3(11,5 \%)$ & $\mathbf{2 1}(\mathbf{3 6 , 8} \%)$ & $30(10,3 \%)$ & $<0,001$ \\
Urgencia-incontinencia & $1(0,7 \%)$ & $0(0 \%)$ & $2(6,1 \%)$ & $1(3,8 \%)$ & $\mathbf{1 2}(\mathbf{2 1 , 1 \% )}$ & $16(5,5 \%)$ & $<0,001$ \\
Dolor & $\mathbf{5 0 ( 3 6 , 2 \% )}$ & $5(13,2 \%)$ & $9(27,3 \%)$ & $5(19,2 \%)$ & $4(7 \%)$ & $73(25 \%)$ & $<0,001$ \\
Retención urinaria & $37(26,8 \%)$ & $1(2,6 \%)$ & $\mathbf{1 8 ( 5 4 , 5 \% )}$ & $4(15,4 \%)$ & $5(8,8 \%)$ & $65(22,3 \%)$ & $<0,001$ \\
$\begin{array}{l}\text { Residuo postmiccional } \\
\text { elevado }\end{array}$ & $\mathbf{3 6 ( \mathbf { 2 6 , 1 } \% )}$ & $3(7,9 \%)$ & $6(18,2 \%)$ & $1(3,8 \%)$ & $0(0 \%)$ & $46(15,8 \%)$ & $<0,001$ \\
\hline
\end{tabular}


técnica) aunque, de modo preocupante, el empleo de Burch por vía retropúbica presenta, en nuestra experiencia, tan sólo un $59 \%$ de pacientes continentes después de los dos años tras la cirugía.

Como ya es bien conocido, se han abandonado las técnicas laparoscópicas al no encontrar ventajas respecto al Burch en términos de continencia $^{12}$. En nuestra experiencia tan sólo $59 \%$ están libres de incontinencia a los dos años. Además tampoco está exenta de complicaciones como la inestabilidad del detrusor de novo, intravesicalización de tackers, alto coste, etc ${ }^{2}$.

Es importante tener en cuenta el diagnóstico preoperatorio de incontinencia urinaria mixta. En nuestra serie existe una asociación entre TVT y los sintomas de urgencia o urgencia-incontinencia postoperatorias. Lamentablemente no disponemos de cuántas de estas pacientes presentaban incontinencia mixta antes de la cirugia, dato importante a la hora de interpretar los resultados ${ }^{15}$. Otra limitación del presente estudio es intrinseca al tipo de análisis realizado. El análisis de supervivencia exige definir el evento (en este caso la aparición de incontinencia) de manera muy precisa (definida de modo binario: continente o incontinente), no permitiendo reflejar qué proporción de mujeres no completamente curadas (consideradas aquí como fracasos de la cirugía) han mejorado su sintomatología y calidad de vida. Es probable que el criterio que hemos definido como fracaso de la cirugía sea muy estricto, presentando por ello tasas de curación por debajo de lo observado en otras series.

\section{CONCLUSIONES}

La técnica con mejores resultados para manejo de incontinencia en nuestra población es el TVT, con la contrapartida de una mayor tasa de urgencia e y urgencia-incontinencia postoperatorias, aunque con una baja probabilidad de componente obstructivo en nuestras manos.

\section{REFERENCIAS}

1. Asociación Española de Urología y Sociedad Española de Ginecología y Obstetricia. Tratado de Incontinencia Urinaria. Luzán Editores, 2006. P. 545

2. Bulent Tiras M, Sendag F, Dilek U, Guner H. Laparoscopic burch colposuspension: comparison of effectiveness of extraperitoneal and transperitoneal techniques. Eur $\mathrm{J}$ Obstet Gynecol Reprod Biol. 2004 Sep 10;116(1):79-84.
3. Tennstedt S; Urinary Incontinence Treatment Network. Design of the Stress Incontinence Surgical Treatment Efficacy Trial (SISTEr). Urology. 2005 Dec;66(6):1213-1217.

4. Bai SW, Sohn WH, Chung DJ, Park JH, Kim SK. Comparison of the efficacy of Burch colposuspension, pubovaginal sling, and tension-free vaginal tape for stress urinary incontinence. Int J Gynaecol Obstet. 2005 Dec;91 (3):246-251.

5. Artibani W. Effective Use Of Surgery In Managing Mixed Incontinence. Europ Journal 2006;5(16):863-865.

6. Albright T, Garlich C, Iglesias C. Complications After Laparoscopic Burch With Hernia Mesh And Surgical Tacks. Obstet Gynecol. 2006;108: 718-720.

7. Morey AF, Medendorp AR, Noller MW, Mora RV, Shandera KC, Foley JP, Rivera LR, Reyna JA, Terry PJ. Transobturador Versus Transabdominal Mid Urethral Slings: A Multi-Institutional Comparison Of Obstructive Voiding Complications. J Urol 2006;175 (3pt1):1014-1017.

8. Darai E, Frobert J.L, Grisard-Anaf M, Lienhart J, Fernandez H, Dubernard G, David-Montefiore E. Functional results after the suburethral sling procedure for urinary stress incontinence: a prospective randomized multicentre study comparing the retropubic and transobturator routes. Eur Urol. 2007 Mar;51(3):795-801.

9. Thakar R, Stanton S. Regular review: management of urinary incontinence in women. BMJ. 2001 Apr 21;322 (7292): 997.

10. Luján Galán M, Páez Borda A, Bustamante Alarma S, Fernández González I, Gago Juan A, Berenguer Sánchez A. Análisis comparativo de diferentes técnicas para la corrección de la incontinencia de stress en la mujer. Revisión de nuestra experiencia. Actas Urol Esp. 1997;21(7):655-661.

11. Kaplan E. L, Meier P. Nonparametric estimation from incomplete observations. J Am Stat Assoc1958;53:457481.

12. Robert M, Farrell SA; Society of Obstetricians and Gynaecologists of Canad. Choice Of Surgery For Stress Incontinence. Int J Gynaecol Obstet. 2006 Oct;95(1):8896.

13. Redondo González E, Luján Galán M, Páez Borda A, Moreno Santurino A, Santos Arrontes D, Berenguer Sánchez A. Análisis comparativo de complicaciones entre las diferentes técnicas empleadas para el tratamiento quirúrgico de la incontinencia urinaria de esfuerzo en nuestro medio. Arch Esp Urol. 2003;56(3):269-275.

14. Brubaker L. Surgical treatment of urinary incontinence in women. Gastroenterology. 2004 Jan;126(1 Suppl 1):S7176.

15. Holmgren C, Nilsson S, Lanner L, Hellberg D. Frequency Of The Novo Urgency In 463 Women Who Had Undergone The Tension-Free Tape (TVT) Procedure For Genuine Stress Urinary Incontinence - A Long Term Follow Up. Eur J Obstet Gynecol Reprod Biol. 2007 May;132(1):121125.

Correspondencia autora: Dra. G. Torres Zambrano Servicio de Urologia. Hospital Universitario de Getafe Ctra. Toledo. Km 12.500 - 28905 Getafe (Madrid) Tel.: 916839360

E-mail autora: gmtorres.hugf@salud.madrid.org Información artículo: Original - Incontinencia femenina Trabajo recibido: octubre 2006 Trabajo aceptado: abril 2007 